\title{
Temporal Visual Field Border
}

\section{Jan Lestak (D) \\ Tomas Lestak \\ Martin Fus \\ Iva Klimesova}

Faculty of Biomedical Engineering, Czech Technical University in Prague, Prague, Czech Republic
Correspondence: Jan Lestak Faculty of Biomedical Engineering, Czech Technical University in Prague, nám. Sítná 3105, Kladno, Prague, 272 0I, Czech Republic

Tel +420602336770

Email lestak@seznam.cz
Purpose: The aim of the study was to determine the physiological temporal visual field border, based on theoretical calculations and by perimetric examination itself.

Materials and Methods: A perimetry test was performed on 15 healthy subjects - seven women (27-30 years old) and eight men (28-46 years old), all of whom had healthy eyes with a visual acuity value of 1.0. The visual field was evaluated using a Medmont M700 with nasal displacement of the fixation point of 40 degrees. In total, 179 examined points of visual field were included. The model of the entry of temporal rays into the eye was created on the basis of the measured biometric values of the eye and with the help of the AD systems AutoCad and SolidWorks. RayViz for SolidWorks was able to simulate the passage of light rays through the model.

Results: The temporal part of the subjective visual field border was up to 110 degrees in all eyes. Modelling of the input rays based on geometric optics in one of the participants in the testing revealed a theoretical temporal boundary of the visual field of 102 degrees.

Conclusion: Theoretical calculations of geometric optics have shown that the temporal boundary of the visual field reaches up to 102 degrees in a healthy individual. By perimetric examination, this limit reached 110 degrees.

Keywords: visual field, temporal visual field border, optimal eye parameters, perimetry

\section{Introduction}

As far back as 1823, Jan Evangelista Purkinje designed a device made of threequarters of a circular band for the examination of the visual field, which could be rotated in front of the examined eye to any position. This allowed examination of the visual field in any meridian. ${ }^{1}$ Purkinje also described the boundaries of the visual field:

My measurements of the width of indirect vision provided the following results: to the outer corner of 100 degrees (with the pupil dilated by belladonna to 115), down to 80 degrees, up to 60 degrees and just as much to the inner corner. ${ }^{2}$

In contemporary Czech ophthalmology textbooks, the extent of the visual field is described only in Heissigerová. The visual field is the part of space that a person perceives when fixing the eye on one point. Temporally, the field has a range of 95, nasally 65, superiorly 60, and inferiorly 70 degrees. $^{3}$ Furthermore, Skorkovská states that the range of the normal visual field extends to more than 90 degrees temporally, to 60 degrees nasally and superiorly, and to about 70 degrees inferiorly. ${ }^{4}$ Various data on the temporal boundary of the visual field can also be found in foreign literature.

Pöppel and Harvey report nasal visual field boundaries of 50 to 60 degrees and temporal up to 90 degrees. $^{5}$ Spector describe peripheral vision reaching up to 100 
degrees temporally and 60 degrees nasally, superiorly, and inferiorly. ${ }^{6}$ Similarly, Heijl et al and Racette et al report a visual field range of more than 90 degrees temporally, 70 degrees inferiorly, and 60 degrees nasally and superiorly. ${ }^{7-9}$ However, one must be aware that these data were obtained in kinetic perimetry.

The reason for performing this study was not only due to the discrepancies within the stated limits, but also as an early reminder of the bicentennial of the construction of the device for the examination of the visual field by J. E. Purkyně. Currently, examination of the visual field is performed by static automatic perimetry. Therefore, the main purpose of this study was to determine its temporal boundaries and verify these conclusions by theoretical modelling of the boundary rays entering the eye from this side.

\section{Materials and Methods}

We included 15 healthy people in the group. Seven were women aged 27-30 and eight were men aged 28-46. Physiological findings in the eyes, including pupil response and visual acuity of 1.0, were determined in all eyes. As it was not possible to assess the correctness of the fixation, the whole group of 15 people consisted of medical staff (physicians and nurses) from the ophthalmology department. Everyone knew the examination using this technology, so we could rule out artificial findings in advance, including incorrect fixation. The examination was performed under natural conditions, without artificial mydriasis.

Examination of the visual field was performed using a Medmont M700 (Medmont Pty Ltd, Australia) instrument using a glaucoma program (fast threshold strategy), where it was possible to supplement the temporal part of

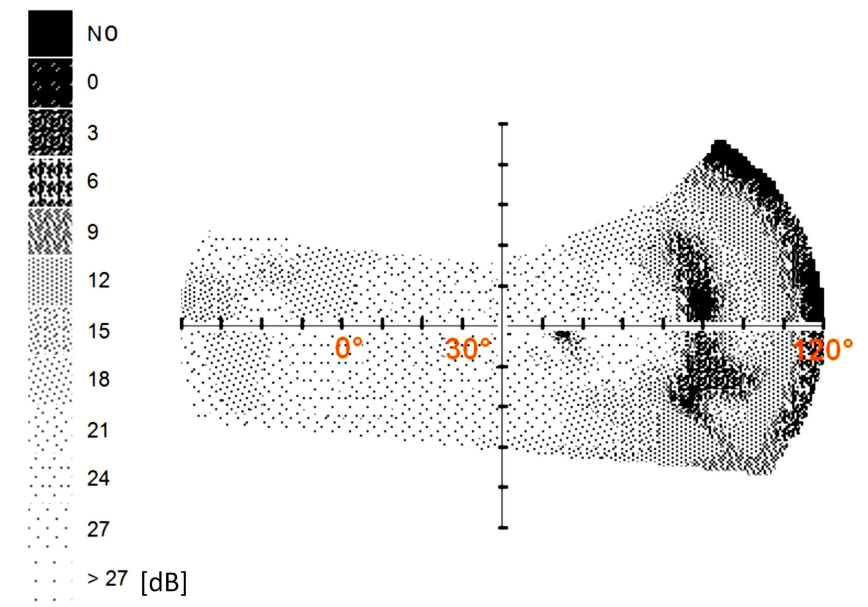

the visual field with additional examination points to a total of 179 , moving the fixation point 40 degrees nasally. The minimum horizontal distance between the two measured points in the edge area was equal to 10 degrees. The result was the possibility of examining the visual field temporally up to 120 degrees (Figure 1).

The model for simulating the passage of light rays through the eye, enabling the theoretical determination of the limiting temporal angle, consisted of five separate parts corresponding to the considered refractive interface (cornea, aqueous humour, lens, vitreous humour, and sclera). The used values of the parameters of the individual elements of the optical model were determined by measuring a specific eye of one of the examined persons. Biometric data (corneal thickness, axial eye length, lens thickness and anterior chamber depth) were measured using a Lenstar instrument (Haag-Streit diagnostics, Switzerland). Measurements of the radius of the anterior and posterior corneal surfaces, ventricular angle, pupil width, and external and internal radii of curvature of the lens were performed using an OCT Avanti RT-Vue XR instrument (Optovue, USA). Measurement of the biometric data and subsequent calculation of the cut-off angle was performed under natural conditions (without artificial mydriasis). Other parameters for modelling (especially refractive indices) were taken from Gullstrand's schematic model of the eye, and the sclera was defined as the "perfect absorber" of the emitted rays. The theoretical range of the visual field was calculated from the visual axis, ie the axis connecting the fovea and the observed point through the nodal points. We proceeded from the simplification that the beam, after entering the

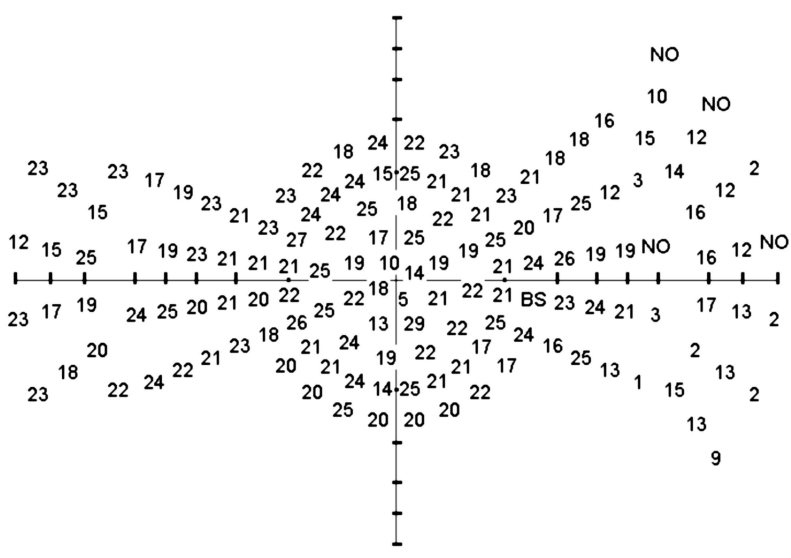

Figure I Temporal border of the visual field with a displacement of the fixation point nasally by 40 degrees. 
eye, passes through four optical surfaces, the anterior and posterior surfaces of the cornea and the anterior and posterior surfaces of the lens, and is limited by the size of the pupil. Furthermore, the sphericity of the anterior and posterior surfaces of the cornea was approximated without considering the decentration of the pupil. This procedure was inspired by the study by $\operatorname{Simson}^{10}$ and Ramasubramanian et al. ${ }^{11}$ We created a model of the entry of temporal rays into the eye based on the measured biometric values of the eye using the CAD systems AutoCad (AutoDesk) and SolidWorks (Dassault Systèmes). RayViz for SolidWorks (Lambda Research Corporation) allowed us to simulate the passage of light rays through an eye model.

\section{Results}

In all the examined eyes, the range of temporally seen points was determined by the perimetric examination to 110 degrees. Figure 1 illustrates the examination of the same eye in which the boundary beam input was modelled (Figure 2). For completeness, we state that the Medmont device detects fixation using the Heijl-Krakau method. By moving the fixation point, the blind spot is incorrectly localized, because the program does not consider the change of fixation (Figure 1).

When modelling the movement of the rays through the eye, it was assumed that the beam originates from an emitter $33 \mathrm{~cm}$ away from the eye, thus mimicking the perimeter examination. By gradually increasing the angle of the incident beam to the visual axis, the boundary of the temporal field of view was determined to be 102 degrees as the largest angle at which light still passed through the lens and hit the retina. The resulting optical model is shown in Figure 2. The image also contains an example of a simulated beam that was still able to pass through the lens. The reflected and absorbed rays are not shown for better clarity. The pupil diameter was determined to be $4.64 \mathrm{~mm}$.

\section{Discussion}

The Medmont M700 perimeter, which is the most widely used in the Czech Republic, examines the visual field temporally when the fixation mark is moved up to 80 degrees, the Octopus perimeter up to 90 degrees, the HFA (Humphrey Field Analyzer) perimeter up to 89 degrees and the Oculus perimeter up to 85 degrees. The neurological program (examination of the entire visual field) is in the range of 0 to 50 degrees for the Medmont, 0 to 75 degrees for the Octopus, 0 to 89 for the HFA and 0 to 85 degrees for the Oculus. Thus, none of the commercially available perimeters in the Czech Republic examines the visual field up to its temporal boundary, so we were interested in which of the above-mentioned data on peripheral boundaries were correct. In order to recognize points located temporarily at an angle of 90 degrees or more, the following conditions must be met:

- The optical media of the eye must receive a peripheral beam incident on the cornea into the pupil.

- The light beam that hits the retina must be sensory processed.

Based on the geometric optics and biometric data of the measured eye, we determined at what angle the incoming rays are still able to fall on the posterior pole of the eye. The rays entering the eye passed at angles of up to 102 degrees. From a sensory point of view, the corresponding peripheral parts of the retina should be functional, because at 90 degrees nasally there are 5000 rods per $\mathrm{mm}^{2}$ and about 500 suppositories per $\mathrm{mm}^{2}$, and this number persists at 100 degrees. In the temporal part at 70 degrees there are 3000 rods per $\mathrm{mm}^{2}$ a 500 rods per $\mathrm{mm}^{2} .^{12}$

The density of rods in the nasal half of the retina is therefore higher than in the temporal half. ${ }^{13}$ The situation is similar with ganglion cells, of which there are $300 \%$ more in the periphery of the nasal retina than in the temporal one. ${ }^{14}$ This means that the required peripheral parts of the retina are not "blind". Modelling of rays entering the eye from the temporal side in pseudophakic eyes for negative dysphotopsia was analysed by Ramasubramanian et al. In their experiments, they showed that rays entering the eye from the temporal side at an angle of 90 degrees pass through optical media through the pupil into the eye. ${ }^{11}$ Our results of temporal boundary measurements showed a range of 110 degrees. These data do not differ significantly from the modelling of the boundary beam input using the biometric parameters of a randomly selected patient. Since the visual field examination is performed under the conditions of illumination of the perimeter dome of 31.5 asb and we do not have the results of the ambient brightness in the biometric measurement of the value, this fact could also play a role in the small difference in the results. The results of the perimetric examination are decisive for us. It is clear that mydriasis broadens the field of view. ${ }^{2}$ All measurements were performed under standard natural conditions (without artificial mydriasis). 


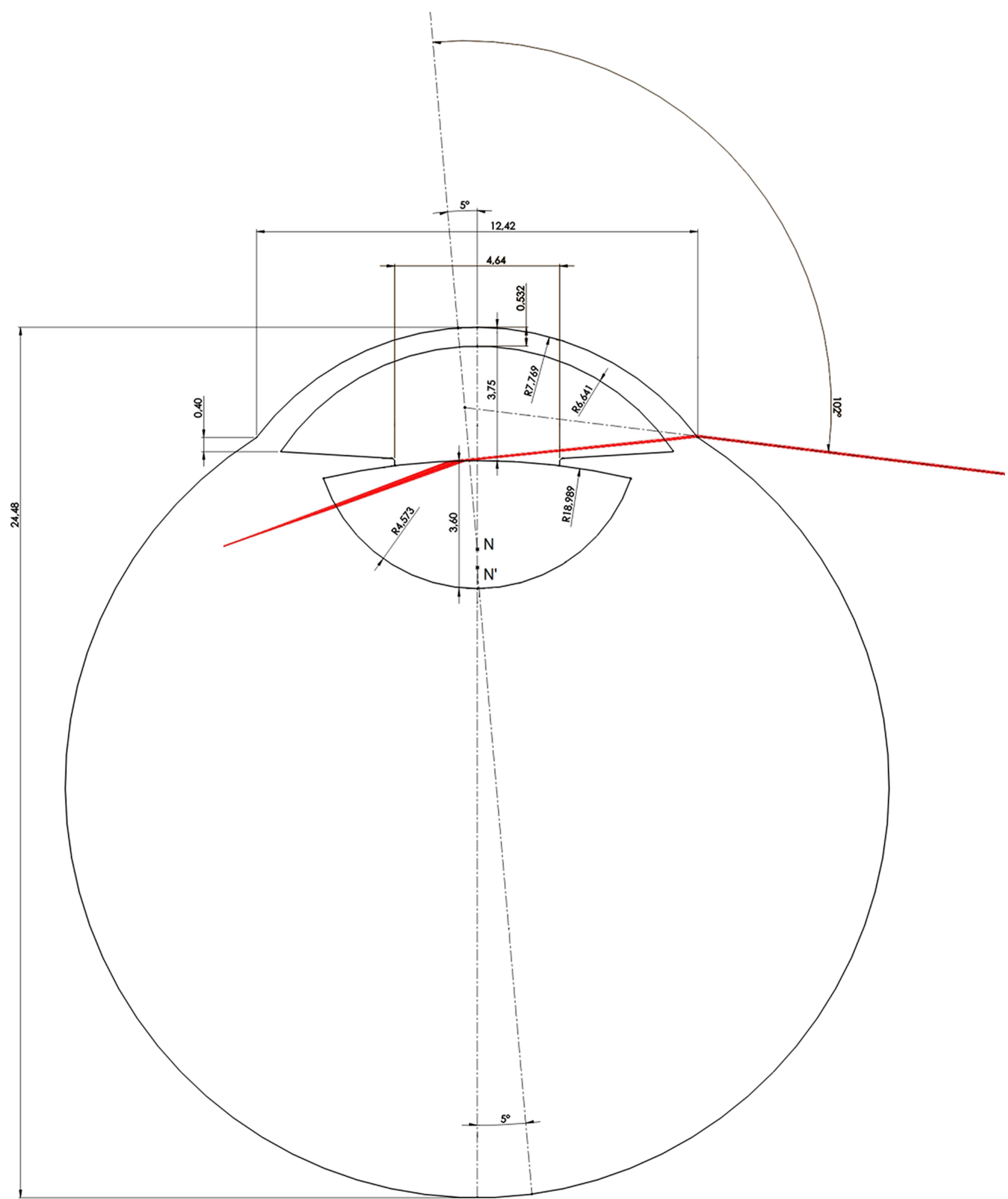

Figure 2 Biometric values of the measured eye. Modelling of the temporal ray at an angle of 102 degrees. The values are in mm and angle degrees. The axis of vision is displaced 5 degrees nasally relative to the axial axis of the eye.

Most textbooks on perimetry recommend from the range of programs for the examination of the visual field those which show its central part (0 to 30 degrees), including neurological affections. ${ }^{7-9}$ However, it is important to examine the entire visual field, which can be evidenced by the claim that if the lesion is located in the visual system, then we should diagnose 
it. It can be deduced from the anatomy of the visual pathway that peripheral changes in the visual field can be observed in peripheral retinal diseases, optic perineuritis, small oppressive peripheral lesions, damage to the anterior calcarine sulcus either unilaterally or bilaterally, and hypertensive glaucoma, especially in their initial stages. Otradovec comments on perimetry:

Due to its high and irreplaceable topical value, examination of the visual field in neuroophthalmological diagnostics is by far the most important functional test. Nevertheless, it is often postponed, perhaps due to unfounded prejudice about its professional, time and equipment requirements. ${ }^{15}$

When we realize that a healthy individual can see up to 110 degrees, then the Medmont perimeter lacks up to 60 degrees temporally in the neurological program of the untested. In ophthalmology, visual field examination is most often used in glaucoma. It occurs just in hypertensive glaucoma that the magnocellular (M) ganglion cells, which are located in the periphery of the retina, are damaged. ${ }^{16,17}$ Similar conclusions are presented by Kerrigan-Baumrind et al. Significant changes in the visual field were associated with a loss of up to $25-35 \%$ of retinal ganglion cells, with $\mathrm{M}$ cells dying preferentially. However, it should be emphasized that the authors used the 30-2 (Humphrey field analyser) program for the examination. ${ }^{18}$

Although in our previous study we did not find a difference in the examination of incipient hypertensive glaucoma using a glaucoma program and a program in the range of 0 to 50 degrees, this does not mean that there will be no significant changes above 50 degrees. ${ }^{19}$

\section{Conclusion}

The theoretical results of geometric optics as well as the results of the perimetric examination showed that the temporal boundaries of the visual field in a healthy individual reach up to 102 and the subjective limit according to perimetry reaches 110 degrees in the whole evaluated group.

\section{Ethics Approval}

This work was carried out according to the Declaration of Helsinki and was approved by the internal ethics committee of the Ophthalmology clinic JL, V Hůrkách 1296/10, 15800 Praha 5 - Nové Butovice, Czech Republic. All healthy participants provided informed consent to take part in this study.

\section{Disclosure}

The authors report no conflicts of interest in this work.

\section{References}

1. Kuthan V. Some contributions of J. E. Purkyně to the visual physiology. Physiol Bohemoslov. 1987;36:255-267.

2. Kurz J. Neuro-O phthalmology Diagnostics. SZN Praha; 1956:1018.

3. Heissigerová J. Ophthalmology. Maxdorf Jessenius; 2018. ISBN 97880-7345-580-4.

4. Skorkovská K. Perimetry. Grada Publishing a.s; 2015. ISBN 978-80247-5282-2.

5. Pöppel E, Harvey LO Jr. Light-difference threshold and subjective brightness in the periphery of the visual field. Psychol Forsch. 1973;36:145-161. doi:10.1007/BF00424967

6. Spector RH. Chapter 16: Visual Fields. In: Clinical Methods: The History, Physical, and Laboratory Examinations. Walker $\mathrm{K}$, Hall WD, Hurst JW, editors. 3rd ed. Boston: Butterworths; 1990. ISBN-10: 0-409-90077-X

7. Heijl A, Patella VM. Essential Perimetry. The Field Analyser Primer. 3rd ed. Carl Zeiss Meditec, Inc; 2002. ISBN 0-9721560-0-3.

8. Heijl A, Patella VM, Bengtssone B. Effective Perimetry: The Field Analyser Primer. 4th ed. Carl Zeiss Meditec, Inc.; 2012. ISBN 0-9884795-0-8.

9. Racette L, Fischer M, Bebie H, Holló G, Johnson CA, Matsumoto C. A Guide to Perimetry and the Octopus Perimeter. 7th ed. Könitz, Switzerland: Haag-Streit AG; 2018. ISBN: 978-3-033-06551-2.

10. Simpson MJ. Mini-review: far peripheral vision. Vision Res. 2017;140:96-105. doi:10.1016/j.visres.2017.08.001

11. Ramasubramanian V, Meyer D, Kollbaum PS, Bradley A. Experimental model of far temporal field negative dysphotopsia generated in Phakic eyes. Invest Ophthalmol Vis Sci. 2020;61(5):24. doi:10.1167/iovs.61.5.24

12. Østerberg G. Topography of the layer of rods and cones in the human retina. Acta Ophthalmol Suppl. 1935;13:1-102.

13. Curcio CA, Sloan KR, Kalina RE, Hendrickson AE. Human photoreceptor topogtraphy. J Comp Neurol. 1990;292:497-523. doi:10.1002/cne. 902920402

14. Curcio CA, Allen KA. Topography of ganglion cells in human retina. J Comp Neurol. 1990;300(1):5-25. PMID: 2229487. doi:10.1002/ cne. 903000103

15. Otradovec J. Klinická Neurooftalmologie. Grada Publishing; 2003. ISBN 80-247-0280-0.

16. Morgan JE, Uchida H, Caprioli J. Retinal ganglion cell death in experimental glaucoma. $\mathrm{Br} \quad J$ Ophthalmol. 2000;84:303-310. doi:10.1136/bjo.84.3.303

17. Shou T, Liu J, Wang W, et al. Differential dendritic shrinkage of $\alpha$ and $\beta$ retinal ganglion cells in cats with chronic glaucoma. Invest Ophthalmol Vis Sci. 2003;44:3005-3010. doi:10.1167/ iovs.02-0620

18. Kerrigan-Baumrind LA, Quigley HA, Pease ME, Kerrigan DF, Mitchell RS. Number of ganglion cells in glaucoma eyes compared with threshold visual field tests in the same persons. Invest Ophthalmol Vis Sci. 2000;41:741-748.

19. Lešták J, Fůs M. Visual field assessment in hypertension glaucoma. Cesk Slov Oftalmol. 2021;77:20-24. doi:10.31348/2021/12 


\section{Publish your work in this journal}

Clinical Ophthalmology is an international, peer-reviewed journal covering all subspecialties within ophthalmology. Key topics include: Optometry; Visual science; Pharmacology and drug therapy in eye diseases; Basic Sciences; Primary and Secondary eye care; Patient Safety and Quality of Care Improvements. This journal is indexed on PubMed
Central and CAS, and is the official journal of The Society of Clinical Ophthalmology (SCO). The manuscript management system is completely online and includes a very quick and fair peer-review system, which is all easy to use. Visit http://www.dovepress.com/ testimonials.php to read real quotes from published authors. 